\title{
Numerical Evidence for Spontaneously Broken Replica Symmetry in 3D Spin Glasses
}

\author{
E. Marinari \\ Dipartimento di Fisica and INFN, Università di Cagliari, Via Ospedale 72, 09100 Cagliari, Italy \\ G. Parisi and J. Ruiz-Lorenzo \\ Dipartimento di Fisica and INFN, Università di Roma La Sapienza, P. A. Moro 2, 00185 Roma, Italy \\ F. Ritort \\ Departamento de Matemáticas, Universitad Carlos III, Butarque 15, Leganés 28911, Madrid, Spain
}

(Received 10 August 1995)

\begin{abstract}
Using numerical simulations of the 3D Ising spin glass we find evidence that spontaneous replica symmetry breaking theory and not the droplet model describes with good accuracy the equilibrium behavior of the system.

PACS numbers: 75.50.Lk, 05.50.+q, 64.60.Cn
\end{abstract}

The behavior of disordered spin models at equilibrium is well understood in the framework of the mean field approximation [1,2]. The main prediction of the mean field approach is the existence of a low-temperature glassy phase. Such a phase is characterized by the existence of many different equilibrium states [spontaneous replica symmetry breaking (SRSB)].

On the other hand, it is possible to define a different consistent theory [3] by starting from the Migdal-Kadanoff approach. We will refer to this approach in the following as the droplet model. Here one expects the equilibrium state to be unique (apart from global inversions in zero magnetic field) and that the most relevant excitations are obtained by reversing large domains of spins (the droplets).

We have two different starting points. One is the infinite range approximation which leads to the replica symmetry breaking picture, and the other is the Kadanoff-Migdal approximation which leads to the droplet model. Although each of the two pictures is correct in its range of validity, we have to establish which qualitatively describes the physics of real three-dimensional spin glasses.

A physical model behaving as a droplet would be reminiscent of a usual phase transition of the Curie type. On the contrary mean-field-like behavior would imply new features. Experimentalists are working hard trying to detect or falsify such behavior, and the question is far from settled [4].

The main result of this work (which continues the investigation started in [5], and follows a long series of Monte Carlo simulations of spin-glass systems [6]) has been to gather new and strong evidence that in three dimensions the SRSB picture (and not the droplet model) describes correctly what is observed in numerical simulations.

Let us start by summarizing the evidence we will present in this Letter and the scheme of our reasoning (for a more detailed exposition of these and more data see Ref. [7]). We will start by showing that the probability distribution of the overlap among two systems at equilibrium, $P(q)$, has a nontrivial structure. $P(q \simeq 0)$ is different from zero, and its shape does not depend on the volume. We will analyze (following a suggestion contained in the third reference of [8]) sample to sample fluctuations of the spin-glass susceptibility, and find that they are incompatible with the droplet model, while their size is very well explained (even in a quantitative manner) by SRSB theory. In order to show that the structure of the different equilibrium states is not compatible with a droplet structure we will compute and analyze equal time correlation functions. From this analysis we deduce the existence of many equilibrium states that cannot be described by a dropletlike structure. On the contrary, we will show that even at a quantitative level SRSB theory explains very well the numerical data.

Further evidence on the inadequacy of the droplet model to describe the 3D spin glasses and support for a SRSB mechanism is provided by analyzing the distribution of overlaps of boxes of side $R, q_{R}(x) \equiv R^{-D} \sum_{y} \sigma_{x+y} \tau_{x+y}$ (where $y$ is an integer vector which takes all the $R^{D}$ values compatible with the conditions $0 \leq y_{\nu}<R$ ) and by discussing the behavior of the box overlap Binder cumulant $g(R, t) \equiv \frac{3}{2}-\left\langle q_{R}^{4}\right\rangle / 2\left\langle q_{R}^{2}\right\rangle^{2}$.

The model we will mainly consider is defined by the simple Edwards-Anderson Hamiltonian on a 3D simple cubic lattice $H \equiv-\sum_{\{i, k\}} \sigma_{i} J_{i, k} \sigma_{j}$, where the sum runs over nearest neighbor couples of sites. The quenched disordered couplings $J$ are distributed according to a Gaussian law. A study of the overlap susceptibility and of the Binder cumulant shows that (under the a priori assumption of the existence of a phase transition at a nonzero temperature with a power law divergence) the transition is located at $T=1.00 \pm 0.05$. In order to check universality of our results we have also studied a model [7] with integer $J= \pm 1$ variables, where each spin is coupled with equal strength to 26 neighboring sites (all the ones contained in a cube of $3^{3}$ sites). The results we discuss are confirmed by our findings about this second model.

We have used an isotropic lattice of linear size $L$, and we have computed the probability distribution $P_{J}(q)$ of the overlap $q \equiv V^{-1} \sum_{i} \sigma_{i} \tau_{i}$ among two thermalized 
configurations $\sigma$ and $\tau$ in a box of volume $V=L^{3}$. We have studied the behavior of the function $P(q)$ averaged over a large number of realizations of the quenched disordered couplings $J$ [i.e., the average over the $J$ random variables of $\left.P_{J}(q)\right]$. We have used a maximum of 2560 samples for the smallest lattice sizes and a minimum of 512 samples for the largest sizes. It was already known (see, for example, [5] and references therein) that $P_{J}(q)$ is nontrivial and has a shape quite similar to the one predicted in the mean field model. Mainly thanks for the use of large computer resources (we have mainly used the APE parallel computer [9], which turns out to be very effective for this kind of problem [7]: we flip about $2 \times 10^{8}$ spins per second on the tower version of the machine) and of the tempering (an annealinglike improved Monte Carlo technique introduced in [10]) we have been able to study systems of larger size than before (up to $14^{3}$ ), and to bring them to thermal equilibrium quite deep in the cold phase. In this case we have equilibrated the system up to distance 14. The tempering method allows one to check thermalization by monitoring the distribution of the temperature values dynamically selected by the system. We have also checked that for each individual sample the function $P(q)$ is symmetric under the exchange $q \leftrightarrow-q$ (this is a very strong thermalization check). We will see that this information is complemented by our dynamical study, where we work on time scales on which we can equilibrate the system on distances up to order 6 . This gives a good control over the fractal geometry of the typical excitations and of their boundaries. The number of points we are considering in an elementary cluster is, in other words, large enough to allow a characterization of the intrinsic geometry. This is what we need in order to distinguish between SRSB theory and Migdal-Kadanoff droplets.

The first crucial comment is that the general form of the function $P(q)$ is size independent in our statistical precision. We stress that the nonzero plateau at low $q$ values, down to $q=0$, is size independent. For example, at $T=0.7$ the Binder cumulant of $q$ is practically independent of the lattice size and it is equal to $0.85 \pm$ 0.01 . This means that the system has a nontrivial structure of equilibrium states with a continuous distribution of the allowed overlap values (even if one should be careful about possible dangerous finite size effects below $T_{c}$ ). By using our measurements of equal time correlation functions we will argue in the following that such states cannot be described by the droplet approach, while they have all features predicted by the SRSB approach.

In this Letter we do not answer a very important question: whether if in the infinite volume limit a lowtemperature phase characterized by the existence of a nonzero order parameter $q_{E A}$ exists. On the lattice volumes we are able to investigate the high $q$ peak of the $P(q)$ is very slowly shifting toward lower $q$ values, even if, as we already said, the shape of the $P(q)$ does not change. The extrapolation to the infinite volume limit appears to be a very delicate issue, and many potential systematic errors (even in the definition of the finite volume $q_{E A}$ ) are involved. Here we will not address in detail this point, and assume that we are working in conditions where the system is effectively frozen in a phase with a nonzero value of $q_{E A}$. A possible scenario [5] of a correlation length diverging exponentially for $T \rightarrow 0$ or of a Kosterlitz-Thouless-like transition would be compatible with this approach, since on our finite lattice we would be measuring properties of a frozen system. It is also important to note that this ambiguity only concerns the behavior of the high $q$ peak of the $P(q)$ (which could tend to $q=0$ on very large lattices), while on the contrary the $P(q)$ for small $q$ values is nontrivial and does not depend on the lattice size.

The agreement with mean field theory becomes quantitative if we study sample to sample fluctuations. Mean field theory tells us how much the function $P_{J}(q)$ for a given realization of the quenched disorder differs from the average. For example, if we consider $\left\langle q^{k}\right\rangle_{J} \equiv \int d q P_{J}(q) q^{k}$, we have in mean field

$$
\overline{\left\langle q^{k}\right\rangle_{J}\left\langle q^{m}\right\rangle_{J}}=\frac{2}{3} \overline{\left\langle q^{k}\right\rangle_{J}} \overline{\left\langle q^{m}\right\rangle_{J}}+\frac{1}{3} \overline{\left\langle q^{k+m}\right\rangle_{J}},
$$

where by the overline we indicate an average over the quenched noise. We have verified that in the low- $T$ region this equality is very well satisfied. For example, for $k=2$ and $m=2$ at $T=0.7$ and $L$ ranging from 4 to 10 the ratio of the left-hand side to the right-hand side of (1) is equal to 1.0 with an error never larger than 0.1 . The relation would be trivially satisfied by a delta function, but this is not our case.

Strictly speaking, the nontriviality of the function $P(q)$ is not in violent contradiction with the droplet model. In the framework of the droplet approach it is always possible to suppose that states where domains that take a finite part of the whole system are reversed have a finite probability. This hypothesis is, however, rather unnatural, and it is definitely wrong in the Kadanoff-Migdal approximation. Moreover, we have already seen that the ability of the SRSB theory to predict quantitatively the fluctuations of the function $P(q)$ is remarkable.

A further argument against the possibility discussed in the last paragraph comes from considering $q-q$ correlation functions restricted to those pairs of configuration which have a small value of $q$. The analysis of such correlation functions, together with the nontriviality of the $P(q)$, constitute an ultimate test of the failure of the droplet model.

More precisely we consider a system of side $L$ and we define the relevant correlation function as

$$
C(x, L)=V^{-1} \overline{\left\langle\sum_{i} \sigma_{i+x} \tau_{i+x} \sigma_{i} \tau_{i}\right\rangle},
$$

where the brackets indicate the thermal average. The droplet model predicts that $C(x, \infty)$ goes to the constant 
value $q_{E A}^{2}$ for large $x$. In the SRSB approach $C(x, \infty) \propto$ $|x|^{-\tilde{\alpha}}$, where $\tilde{\alpha}$ is an exponent which has been computed in less than six dimensions for the $q=0$ correlation functions [11].

We have studied this problem by considering large systems, with $L=64$. In this case we are always very far from equilibrium. We have run numerical simulations starting from two random configurations selected independently (for four realizations of the quenched couplings). We have verified that $q^{2}$ stays small in the whole run so that the difference in the initial configurations, for not too large times, enforces the condition $q \approx 0$. Eventually in a finite system global equilibrium will be reached and $q$ will become of order 1 . However, if we let $L \rightarrow \infty$ first, we can use this approach to study the equilibrium value of the correlation function with the constraint of having zero overlap. So, we practically never impose the constraint, but just check that our run is short enough (even if very long) not to create a nonzero overlap.

In order to do that we consider the time dependent equal time correlation function at time $t$

$$
G(x, t)=V^{-1} \overline{\sum_{i}\left\langle\sigma_{i+x} \tau_{i+x} \sigma_{i} \tau_{i}\right\rangle_{t}},
$$

where the average is done at time $t$; i.e., after $t$ Monte Carlo cycles after the random start. We find that for large times $t$ the correlation function $G(x, t)$ is essentially different from zero for distances not too much larger than a dynamic correlation length $\xi(t)$ which increases (and maybe diverges) with time. Our numerical data are well represented with the functional form

$$
G(x, t)=\frac{A(T)}{x^{\alpha}} \exp \left\{-\left(\frac{x}{\xi(T, t)}\right)^{\delta}\right\},
$$

where we have defined $\xi(T, t) \equiv B(T) t^{\lambda(T)}$. In the whole range of distances $1 \leq x \leq 8$ for Monte Carlo times which range from $10^{2}$ to $10^{6}$ full lattice sweeps and a large range of temperatures $T<T_{c}$ (we have done measurements at different temperatures, down to $T_{\min } \simeq 0.3 T_{c}$ ) we get good fits. The exponents $\alpha$ and $\delta$ are weakly dependent on $T$. For example, at $T=0.70$ we get the best values $\alpha=0.50 \pm 0.02$ and $\delta=1.48 \pm 0.02$. The correlation length exponent $\lambda(T)$ is approximately given by $0.16 T$. Such power law growth of the correlation length was already observed by Rieger [12]. In order to study the limit $t \rightarrow \infty$ in a safe way it is even better to avoid global fits and to fit the data at fixed distance $x$ as

$$
G(x, t)=\tilde{G}_{\infty}(x) \exp \left\{-A(x) t^{-\lambda(T)}\right\} .
$$

In this way the extrapolation to infinite time (with the self-implemented constraint of $q=0$ always satisfied) is performed in a very safe way. We plot in Fig. 1 the correlations $\tilde{G}_{\infty}(x)$ (computed at $T=0.7$ ) as a function of the distance in double logarithmic scale.

We have also computed the same quantities by using a different temperature scale. In this second numerical

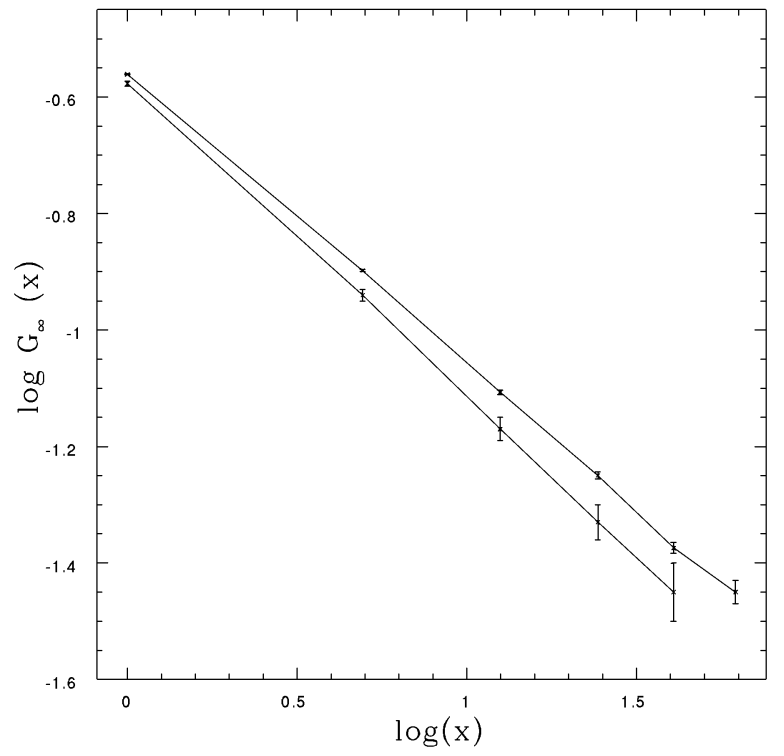

FIG. 1. $\log \left[\tilde{G}_{\infty}(x)\right] \log (x)$, where $\log$ is the natural logarithm. The upper line is from cooling, the lower one from the normal dynamics (see the text for details).

experiment we slowly cool down the system from $T=$ $1.5>T_{c}$ to the final temperature. To perform the cooling we use a number of steps proportional to $t$, the waiting time we want to look at the correlation function. After that the system evolves at the fixed temperature of interest $T$ for $t$ more time steps before measurement. In this way one can obtain a much better equilibration. As a matter of principle, in this case one does not expect a pure power law but a combination of different powers generated by different temperature contributions. However, a fit similar to the previous one (5) works very well with a slightly larger value of $\lambda$. At $T=0.7$ one obtains the results shown in Fig. 1. The data obtained with the two techniques behave in a very similar way. The $t=\infty$ data are well described by a power decay $x^{-\alpha}$ with $\alpha=0.50 \pm$ 0.03 , as predicted by the replica theory and in variance with the droplet model predictions.

The value of the correlation function at distance 1 is particularly interesting. Indeed in the model with Gaussian quenched disorder one can easily prove by a simple integration by part that $E=-\beta[1-C(1)]$, where $E$ is the energy per link and $C(x)$ is the overlap correlation function of the fully equilibrated system (i.e., summed over different ergodic components). Only if replica symmetry is broken does it differ by our correlation function $G$ where the two replicas have been kept (by their own will) in two different ergodic components with zero mutual overlap. The energy can be computed with high accuracy and from its value we can deduce $C(1)$. The equality

$$
C(1)=\tilde{G}_{\infty}(1)
$$

should be violated as soon as replica symmetry is broken, in the same way in which the equality $E=-\beta(1-$ 
$\left.q_{E A}^{2}\right) / 2$ is violated in the Sherrington-Kirkpatrick model at low temperature.

The value of the energy per link is very well fitted by the form $E_{\infty}+A t^{-\Delta(T)}$. The exponent $\Delta(T)$ turns out to be quite large; i.e., we find $\Delta(T) \simeq 0.44 T$, so that it is not difficult to extrapolate the value of the energy to infinite time. If we use the computation of the interface energy done by using SRSB theory [13], we expect that $\Delta(T)=2.5 \lambda(T)$, which is very well satisfied by our data.

While we find that the equality (6) is correct above and at the critical temperature (with less than a relative $1 \%$ error), it is definitely violated below $T_{c}$; at $T=0.7$ we find $C(1)=0.612 \pm 0.001$ and $\tilde{G}_{\infty}(1)=0.56 \pm 0.01$, at $T=$ 0.35 we find $C(1)=0.802 \pm 0.001$ and $\tilde{G}_{\infty}(1)=0.67 \pm$ 0.01 . The failure of the equality (6) implies the existence of different ergodic components. The $q-q$ correlation function depends on the choice of the component, in agreement with the main prediction of the SRSB theory.

As final evidence we discuss the value of $q_{R}(x)$. We evaluate the probability distribution $P_{R}\left(q_{R}\right)$. In the mean field SRSB limit the function $P_{R}\left(q_{R}\right)$ is Gaussian, but in a finite (not too large) number of dimensions it is quite natural to expect deviations from the Gaussian limit.

On the contrary in the droplet model the function $P_{R}\left(q_{R}\right)$ should have two peaks at $q_{R} \approx q_{E A}$, and should become the sum of two delta functions in the limit $R \rightarrow \infty$ (at least for $R \ll L$ ). Indeed here the quantity $q_{R}$ is different from $q_{E A}$ with a probability that goes to zero as a power of $R$.

We have measured the Binder cumulant $g(R, t)$ after $t$ Monte Carlo steps. At a given temperature we expect that

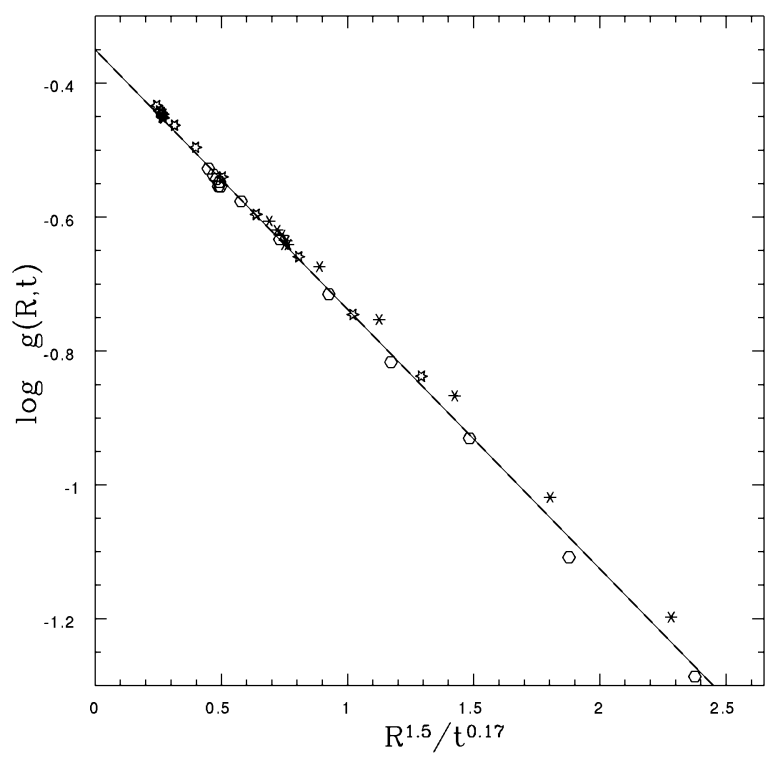

FIG. 2. The logarithm of the Binder cumulant for the box overlap versus rescaled ratio of time and distance. Stars are for $R=2$, hexagons are for $R=3$, and asterisks for $R=4$. The straight line is only to guide the eye. for large $R$ the data will collapse as

$$
g(R, t)=f\left(\left(R \xi(t)^{-1}\right)^{\delta}\right) .
$$

In Fig. 2 we show data for $T=0.7$ from $R=3$ and 4. The scaling law we are proposing works very well. The Binder cumulant extrapolates to something definitely different from 1, which would be the prediction of the droplet model, since in that case the distribution should be asymptotically a pair of delta functions. The data obtained with the alternative temperature scheduling, by relatively slow cooling, give similar results for the extrapolated value of the Binder cumulant.

We can summarize by saying that none of our findings gives support to the predictions of the droplet model, while the broken replica approach is able to predict qualitatively (and in a few cases even quantitatively) the behavior of the 3D spin-glass systems.

We thank J.P. Bouchaud for pointing out to us the potential relevance of local overlaps, and the APE group for continuous assistance and support.

[1] G. Parisi, Phys. Rev. Lett. 43, 1754 (1979); J. Phys. A 13, 1101 (1980); 13, 1887 (1980); 13, L115 (1980); Phys. Rev. Lett. 50, 1946 (1983).

[2] M. Mezard, G. Parisi, and M. Virasoro, Spin Glass Theory and Beyond (World Scientific, Singapore, 1987).

[3] W. L. McMillan, J. Phys. C 17, 3179 (1984); A. J. Bray and M. A. Moore, in Heidelberg Colloquium on Glassy Dynamics, edited by J.L. Van Hemmen and I. Morgenstern (Springer-Verlag, Heidelberg, 1986), p. 121; D. S. Fisher and D. A. Huse, Phys. Rev. Lett. 56, 1601 (1986); Phys. Rev. B 38, 386 (1988).

[4] Recent Progress in Random Magnets, edited by D.H. Ryan (World Scientific, Singapore, 1992).

[5] E. Marinari, G. Parisi, and F. Ritort, J. Phys. A 27, 2687 (1994).

[6] R. Bhatt and A. Young, Phys. Rev. Lett. 54, 924 (1985); Phys. Rev. B 37, 5606 (1988); A. Ogielski and I. Morgenstern, Phys. Rev. Lett. 54, 928 (1985); Phys. Rev. B 32, 7384 (1985); N. Kawashima and A. Young, Report No. cond-mat/9510009.

[7] E. Marinari, G. Parisi, F. Ritort, and J. Ruiz-Lorenzo (to be published).

[8] S. Caracciolo, G. Parisi, S. Patarnello, and N. Sourlas, Europhys. Lett. 11, 783 (1990); J. Phys. (Paris) 51, 1877 (1990); D. Huse and D. Fisher, J. Phys. I (France) 1, 621 (1991); S. Caracciolo, G. Parisi, S. Patarnello, and N. Sourlas, J. Phys. I (France) 1, 627 (1991).

[9] C. Battista et al., Int. J. High Speed Comp. 5, 637 (1993).

[10] E. Marinari and G. Parisi, Europhys. Lett. 19, 451 (1992).

[11] C. de Dominicis, I. Kondor, and T. Temesvári, Int. J. Mod. Phys. B 7, 986 (1993).

[12] H. Rieger, in Annual Reviews of Computational Physics II (World Scientific, Singapore, 1995), p. 295.

[13] S. Franz, G. Parisi, and M. Virasoro, J. Phys. I (France) 2, 1869 (1992). 